\title{
Occurrence of homobaric and heterobaric leaves in two forest types of southern Brazil
}

\author{
Maria Regina Torres Boeger ${ }^{\star *}$, Maiara Matilde Silva', Guilherme Nogueira', Allan Alvarenga' \\ and Suellen Silva Pereto'
}

Received: February 29, 2016

Accepted: May 4, 2016

\begin{abstract}
In ombrophilous forests, light stratification provokes different adjustments by plants for better use of the environmental conditions of each stratum. Among the morphological traits that vary with strata, the presence of bundle sheath extensions (BSEs) is related to water transport, photosynthesis, and leaf mechanical support and classifies leaves as homobaric or heterobaric. This study analyzed the proportion of these types of leaves in a Lowland Ombrophilous Dense Forest (LLODF) and a Mixed Ombrophilous Forest (MOF), and among the strata of each forest type. The morphological leaf traits of 89 LLODF tree species and $57 \mathrm{MOF}$ tree species were examined. The proportion of homobaric and heterobaric leaves did not differ between forests. However, in both forest types, the distribution of species with heterobaric or homobaric leaves depended on strata, with heterobaric species occurring mainly in higher strata, and homobaric species in lower strata. Thus, light stratification acts as an ecological filter on the composition of the vegetation of these forests, favoring heterobaric species in places with higher light intensity and temperature, such as the highest strata of canopy. On the other hand, homobaric species are more frequent in lower strata, where light is less available and humidity higher.
\end{abstract}

Keywords: bundle sheath extension, leaf morphological traits, light stratification, Lowland Ombrophilous Dense Forest, Mixed Ombrophilous Forest

\section{Introduction}

Environmental factors affect the growth and survival of plants (Valladares \& Niinemets 2008) and influence their internal organization (Dickson 2000). In forests, variation in abiotic features along vertical stratification provokes different adjustments by plants for better use of the environmental conditions of each stratum (Valladares \& Niinemets 2008; Niinemets 2010; Inoue et al. 2015). Such adjustments can be morphological, physiological, and/or phenological. Among such morphological traits, the presence of bundle sheath extensions (BSEs) is related to water transport (Zwieniecki et al. 2007), photosynthesis (Pieruschka et al. 2010), and leaf mechanical support (Turner 1994).

Bundle-sheath extensions (BSEs) are formed by parenchyma or sclerenchyma cells that extend from the vascular bundle to both sides of the leaf epidermis (Karabourniotis et al. 2000; Nikolopoulos et al. 2002). Leaves are classified as homobaric or heterobaric depending on the condition of the BSEs (Kenzo et al. 2007). The former lack, or have incomplete, BSEs and their mesophyll is

\footnotetext{
${ }^{1}$ Pós-Graduação em Ecologia e Conservação, Universidade Federal do Paraná, P.O. Box 19031, 81531-990, Curitiba, PR, Brazil

* Corresponding author: mrtboeger@gmail.com
} 
more homogeneous. The latter have complete BSEs and their mesophyll is divided into several photosynthetic compartments (Karabourniotis et al. 2000; Nikolopoulos et al. 2002; Kenzo et al. 2007). Homobaric leaves have a continuous mesophyll (Terashima 1992).

Such structural differences are reflected in the functional proprieties of these leaf types (Kenzo et al. 2007; Pieruschka et al. 2010; Lynch et al. 2012; Inoue et al. 2015). The presence of BSEs can protect leaf lamina from hydric stress and increase light absorption and mechanical support (Terashima 1992; Karabourniotis 1998; Nikolopoulos et al. 2002; Rhizopoulou \& Psaras 2003). Yet in homobaric leaves, gas diffusion in the mesophyll can be more efficient due the absence of BSEs (Pieruschka et al. 2006). Also, BSEs have been linked to light distribution within the mesophyll, allowing investments in thicker and, consequently, smaller leaves (Nikolopoulos et al. 2002).

Despite the light heterogeneity that characterizes Brazilian forests, the occurrence of heterobaric leaves in many plant formations of these biomes and their relation to light gradients is still poorly studied. This study reports on the morpho-anatomical traits of tree species from a Lowland Ombrophilous Dense Forest and a Mixed Ombrophilous Forest in order to investigate the presence and proportion of homobaric and heterobaric leaves in both forest types. Our hypotheses are: a) the frequency of heterobaric leaves is similar in the two studied forest types since both experience similar environmental conditions (annual precipitation and light stratification), independent of their floral composition; b) the distribution and frequency of homobaric and heterobaric leaves vary among different forest strata in response to light stratification, with heterobaric leaves occurring mainly in upper strata and c) leaf type is dependent upon micro-environmental features more so than taxonomic group, as represented by families and/or genus.

\section{Materials and methods}

This study was based on leaf morphological data collected during previous studies in two forest sites: a Lowland Ombrophilous Dense Forest (LLODF), located at Volta Velha
Reserve ( $\left.26^{\circ} 04^{\prime} \mathrm{S}, 48^{\circ} 38^{\prime} \mathrm{W}\right)$, within the city of Itapoá, SC (a detailed description can be found in Boeger et al. 2004); and a Mixed Ombrophilous Forest (MOF), located in the Botanical Garden Francisca Maria Garfunkel Rischbieter ( $\left.25^{\circ} 23^{\prime} 10^{\prime \prime} \mathrm{S}, 49^{\circ} 12^{\prime} 58^{\prime \prime} \mathrm{W}\right)$, within the boundaries of the city of Curitiba, PR (for more details, see Silveira et al. 2015). The environmental characteristics of each forest type are summarized in Tab. 1 . All species included in this study were selected according to two criteria: 1) higher values of importance based on a previous phytosociological survey and 2) the presence of at least three individuals in the forest type. All specimens of collected from LLODF were deposited in UPCB (Herbarium of Department de Botany, UFPR, Curitiba, PR) and specimens from MOF were deposited in MBM (Herbarium of Municipal Botanical Museum, Curitiba, PR).

Morphological data, such as leaf area, leaf thickness, and the presence of homobaric and heterobaric leaves, were collected from 89 LLODF (Tab. 2) and 57 MOF tree species (Tab. 3). Leaves with complete BSEs were classified as heterobaric while leaves with incomplete and/or no BSEs were classified as homobaric leaves, according to Kenzo et al. (2007). The nomenclature and taxonomic classification of each species were checked against International Plant Names Index (www.ipni.org).

All species were classified according to their occurrence in four strata. Species of LLODF were classified into: Stratum 1, <5 m; Stratum 2, 5 - 9.99 m; Stratum 3, 10 - 14.99 m and Stratum 4, >15 m. Species of MOF were classified into: Stratum 1, <7 m; Stratum 2, 7 - 14.99 m; Stratum 3, 15 $26 \mathrm{~m}$ and Stratum 4, >26 m. In MOF, photosynthetically active radiation (PAR) was $28.2 \pm 3.46 \mu \mathrm{mol} \mathrm{s}^{-1} \mathrm{~m}^{-2}(2.3 \%$ irradiance) in Stratum 1; $36.2 \pm 24.3 \mu \mathrm{mol} \mathrm{s}^{-1} \mathrm{~m}^{-2}(3.8 \%$ irradiance) in Stratum 2; $73.6 \pm 22.8 \mu \mathrm{mol} \mathrm{s}^{-1} \mathrm{~m}^{-2}(10 \%$ irradiance) in Stratum 3 and $744.3 \pm 68.4 \mu \mathrm{mol} \mathrm{s}^{-1} \mathrm{~m}^{-2}(100 \%$ irradiance) in Stratum 4 (canopy). In LLODF, PAR was 55.2 $\pm 25.8 \mu \mathrm{mol} \mathrm{s}^{-1} \mathrm{~m}^{-2}$ (4.3\% irradiance) in Stratum 1; $297.4 \pm$ $56.1 \mu \mathrm{mol} \mathrm{s}^{-1} \mathrm{~m}^{-2}$ (23\% irradiance) in Stratum 2; $480.5 \pm 91.1$ $\mu \mathrm{mol} \mathrm{s}{ }^{-1} \mathrm{~m}^{-2}$ (37.3\% irradiance) in Stratum 3 and $1286.8 \pm$ $79.9 \mu \mathrm{mol} \mathrm{s}^{-1} \mathrm{~m}^{-2}$ (100\% irradiance) in Stratum 4 (canopy).

The mean values for the distinct strata of each forest type were compared through One-way ANOVA followed by Tukey test. Leaf area and leaf thickness of homobaric and

Table 1. Environmental features of studied sites. Legend: LLODF - Lowland Ombrophilous Dense Forest; MOF: Mixed Ombrophilous Forest. Climate type according to Köppen classification.

\begin{tabular}{|l|l|l|}
\hline Feature & \multicolumn{1}{|c|}{ LLODF } & \multicolumn{1}{c|}{ MOF } \\
\hline Average moisture $(\%)$ & 85 & 81 \\
\hline Annual precipitation $(\mathrm{mm})$ & 2170 & 1662 \\
\hline Soil type & $\begin{array}{l}\text { Spodosol no hidromorphic. with moderate sandy } \\
\text { texture and low concentrations of exchangeable } \\
\text { cations. }\end{array}$ & $\begin{array}{l}\text { Cambisoil humic aluminic and gleisolic typical } \\
\text { clay soil. }\end{array}$ \\
\hline Altitude $(\mathrm{m})$ & 9 & 947 \\
\hline Mean temperature $\left({ }^{\circ} \mathrm{C}\right)$ & 20.3 & 17.9 \\
\hline Climate type & $\mathrm{Cfb}$ & $\mathrm{Cfb}$ \\
\hline
\end{tabular}


Maria Regina Torres Boeger, Maiara Matilde Silva, Guilherme Nogueira, Allan Alvarenga and Suellen Silva Pereto

Table 2. Presence and absence of bundle sheath extension (BSE) on tree species from Lowland Ombrophilous Dense Forest, by stratum.

\begin{tabular}{|c|c|c|c|}
\hline Family & Species & Stratum & BSE \\
\hline Anacardiaceae & Tapirira guianensis Aubl. & 3 & Absent \\
\hline \multirow[t]{3}{*}{ Annonaceae } & Annona cacans E. Warming & 4 & Absent \\
\hline & Guatteria australis A. St. Hil. & 1 & Absent \\
\hline & Xylopia brasiliensis Spreng. & 3 & Present \\
\hline \multirow[t]{4}{*}{ Aquifoliaceae } & Ilex dumosa Reissek & 2 & Absent \\
\hline & Ilex integerrima Reissek & 2 & Absent \\
\hline & Ilex pseudobuxus Reissek & 2 & Absent \\
\hline & Ilex theezans Mart. & 2 & Absent \\
\hline Araliaceae & Oreopanax capitatus Decne. \& Planch. & 2 & Absent \\
\hline Burseraceae & Protium kleinii Cuatrec. & 2 & Present \\
\hline Calophyllaceae & Calophyllum brasiliense Cambess. & 1 & Absent \\
\hline Celastraceae & Maytenus robusta Reissek & 2 & Absent \\
\hline Clethraceae & Clethra scabra Pers. & 3 & Present \\
\hline \multirow[t]{2}{*}{ Clusiaceae } & Clusia parviflora Engl. & 2 & Absent \\
\hline & Garcinia gardneriana Planch. \& Triana & 1 & Absent \\
\hline Cunoniaceae & Weinmannia paulliniifolia Pohl ex Ser. & 3 & Absent \\
\hline Elaeocarpaceae & Sloanea guianensis Benth. & 2 & Present \\
\hline Erytroxilaceae & Erythroxylum vaccinifolium Mart. & 1 & Absent \\
\hline \multirow[t]{4}{*}{ Euphorbiaceae } & Alchornea triplinervia Müll. Arg. & 2 & Present \\
\hline & Aparisthmium cordatum (A. Juss.) Baill. & 2 & Absent \\
\hline & Maprounea guianensis Aubl. & 2 & Absent \\
\hline & Pera glabrata ex Baill. & 2 & Absent \\
\hline \multirow[t]{4}{*}{ Fabaceae } & Andira anthelminthica Benth. & 2 & Present \\
\hline & Copaifera trapezifolia Hayne & 3 & Absent \\
\hline & Ormosia arborea Harms & 1 & Absent \\
\hline & Pithecellobium langsdorffii Benth. & 2 & Absent \\
\hline \multirow[t]{13}{*}{ Lauraceae } & Aiouea saligna Meisn. & 3 & Present \\
\hline & Aniba firmula (Nees \& Mart.) Mez & 2 & Present \\
\hline & Endlicheria paniculata (Spreng.) J.F.Macbr. & 1 & Absent \\
\hline & Nectandra grandiflora Nees \& Mart. & 2 & Present \\
\hline & Nectandra megapotamica $\mathrm{Mez}$ & 3 & Present \\
\hline & Nectandra oppositifolia Nees \& Mart. & 4 & Present \\
\hline & Ocotea aciphylla Mez & 2 & Present \\
\hline & Ocotea dispersa (Nees \& Mart.) Mez & 2 & Present \\
\hline & Ocotea elegans $\mathrm{Mez}$ & 2 & Present \\
\hline & Ocotea glaziovii Mez & 2 & Present \\
\hline & Ocotea odorifera (Vell.) Rohwer & 2 & Present \\
\hline & Ocotea pulchella Mart. & 2 & Present \\
\hline & Ocotea pulchra (Ekman \& Schmidt) Alain & 4 & Present \\
\hline Malpighiaceae & Byrsonima ligustrifolia A. Juss. & 2 & Absent \\
\hline \multirow[t]{5}{*}{ Melastomataceae } & Miconia cabucu Hoehne & 3 & Absent \\
\hline & Miconia cubatanensis Hoehne & 1 & Absent \\
\hline & Miconia hymenonervia (Raddi) Cogn. & 1 & Absent \\
\hline & Miconia sellowiana Naudin & 1 & Absent \\
\hline & Mouriri chamissoana Cogn. & 2 & Absent \\
\hline Meliaceae & Cabralea canjerana (Vell.) Mart. & 1 & Absent \\
\hline Meliaceae & Guarea macrophylla Vahl & 2 & Absent \\
\hline Monimiaceae & Mollinedia uleana Perkins & 1 & Absent \\
\hline Myristicaceae & Virola oleifera (Schott) A.C.Sm. & 2 & Absent \\
\hline \multirow[t]{7}{*}{ Myrtaceae } & Blepharocalyx salicifolius (Kunth.) O.Berg & 2 & Absent \\
\hline & Calyptranthes concinna DC. & 2 & Absent \\
\hline & Calyptranthes lucida Mart. ex DC. & 1 & Absent \\
\hline & Campomanesia guaviroba (DC.) Kiaersk. & 3 & Present \\
\hline & Eugenia cerasiflora Kurz. & 2 & Absent \\
\hline & Eugenia obovata Wall. ex Duthie & 2 & Absent \\
\hline & Eugenia subavenia O.Berg & 3 & Absent \\
\hline
\end{tabular}




\section{Occurrence of homobaric and heterobaric leaves in two forest types of southern Brazil}

Table 2. Cont.

\begin{tabular}{|c|c|c|c|}
\hline Family & Species & Stratum & BSE \\
\hline \multirow[t]{11}{*}{ Myrtaceae } & Eugenia tristis D.Legrand & 1 & Absent \\
\hline & Eugenia umbelliflora O.Berg & 2 & Absent \\
\hline & Gomidesia affinis (Cambess) D.Legrand & 2 & Absent \\
\hline & Gomidesia schaueriana O.Berg & 1 & Present \\
\hline & Marlierea eugeniopsoides (Kausel \& D.Legrand) D.Legrand & 1 & Absent \\
\hline & Marlierea reitzii D.Legrand & 2 & Absent \\
\hline & Myrceugenia campestris (D.C.) D.Legrand \& Kausel & 2 & Absent \\
\hline & Myrceugenia reitzii D.Legrand \& Kausel & 2 & Absent \\
\hline & Myrcia acuminatissima Hieron. & 2 & Absent \\
\hline & Myrcia fallax DC. & 2 & Present \\
\hline & Psidium cattleyanum Sabine & 3 & Absent \\
\hline Ochnaceae & Ouratea parviflora Engl. & 1 & Absent \\
\hline Olacaceae & Heisteria silvianii Schwake & 2 & Absent \\
\hline Olacaceae & Tetrastylidium grandifolium (Baill.) Sleumer & 2 & Absent \\
\hline Pentaphylacaceae & Ternstroemia brasiliensis Cambess. & 3 & Absent \\
\hline Phyllantaceae & Hieronyma alchorneoides Allem. & 4 & Present \\
\hline Polygonaceae & Coccoloba warmingii Meisn. & 4 & Present \\
\hline \multirow[t]{3}{*}{ Primulaceae } & Conomorpha peruviana A.DC. & 1 & Absent \\
\hline & Rapanea ferruginea $\mathrm{Mez}$ & 4 & Absent \\
\hline & Rapanea venosa Elmer & 2 & Absent \\
\hline Rosaceae & Prunus sellowii Koehne & 2 & Absent \\
\hline \multirow[t]{3}{*}{ Rubiaceae } & Amaioua guianensis Aubl. & 2 & Present \\
\hline & Faramea marginata Mart. & 1 & Absent \\
\hline & Rudgea villiflora K.Schum. ex Standl. & 1 & Absent \\
\hline Rutacea & Esenbeckia grandiflora Mart. & 1 & Absent \\
\hline \multirow[t]{2}{*}{ Sapindaceae } & Cupania oblongifolia Turcz. & 2 & Absent \\
\hline & Matayba guianensis Aubl. & 1 & Absent \\
\hline \multirow[t]{3}{*}{ Sapotaceae } & Manilkara subsericea (Mart.) Dubard & 3 & Absent \\
\hline & Pouteria beaurepairei (Glaz. \& Raunk) Baehni & 2 & Absent \\
\hline & Pouteria venosa (Mart.) Baehni & 2 & Absent \\
\hline Solanaceae & Solanum inaequale C. Presl & 2 & absent \\
\hline Styracaceae & Styrax glabratus Warb. & 3 & Absent \\
\hline Winteraceae & Drimys brasiliensis Miers. & 2 & Absent \\
\hline
\end{tabular}

Table 3. Presence and absence of bundle sheath extension (BSE) on tree species from Mixed Ombrophilous Forest, by stratum.

\begin{tabular}{|c|c|c|c|}
\hline Family & Species & Stratum & BSE \\
\hline \multirow[t]{2}{*}{ Anacardinaceae } & Lithraea molleoides (Vell.) Engl. & 2 & Absent \\
\hline & Schinus terebinthifolius Raddi & 2 & Present \\
\hline Aquifoliaceae & Ilex paraguariensis A.St.-Hil. & 1 & Absent \\
\hline Araucariaceae & Araucaria angustifolia (Bertol.) Kuntze & 4 & Absent \\
\hline Asteraceae & Gochnatia polymorpha (Less.) Cabrera & 2 & Present \\
\hline Bignoniaceae & Jacaranda puberula Cham. & 1 & Absent \\
\hline Canellaceae & Capsicodendron dinisii (Schwacke) Occhioni & 3 & Absent \\
\hline Cannabaceae & Celtis iguanaea (Jacq.) Sarg. & 1 & Absent \\
\hline Cardiopteridaceae & Citronella paniculata (Mart.) R.A.Howard & 2 & Absent \\
\hline Celastraceae & Maytenus ilicifolia Mart. ex Reissek & 1 & Absent \\
\hline Elaeocarpaceae & Sloanea monosperma Benth. & 1 & Present \\
\hline \multirow[t]{2}{*}{ Euphorbiaceae } & Sebastiania brasiliensis Spreng. & 1 & Absent \\
\hline & Sebastiania commersoniana (Baill.) L.B.Sm. \& Downs & 1 & Absent \\
\hline \multirow[t]{4}{*}{ Fabaceae } & Dalbergia brasiliensis Vogel & 2 & Absent \\
\hline & Erythrina falcata Benth. & 3 & Present \\
\hline & Inga marginata Willd. & 2 & Absent \\
\hline & Lonchocarpus muehlbergianus Hassl. & 3 & Present \\
\hline Lamiaceae & Vitex megapotamica (Spreng.) Moldenke & 2 & Present \\
\hline \multirow[t]{2}{*}{ Lauraceae } & Nectandra megapotamica $\mathrm{Mez}$ & 1 & Present \\
\hline & Ocotea porosa (Nees) Angely & 3 & Present \\
\hline
\end{tabular}


Table 3. Cont.

\begin{tabular}{|c|c|c|c|}
\hline Family & Species & Stratum & BSE \\
\hline Lauraceae & Ocotea puberula Nees & 2 & Present \\
\hline Lythraceae & Lafoensia pacari A.St.-Hil. & 2 & Absent \\
\hline Malvaceae & Luehea divaricate Mart. & 2 & Present \\
\hline \multirow[t]{2}{*}{ Melastomataceae } & Miconia sellowiana Naudin & 1 & Absent \\
\hline & Tibouchina sellowiana Cogn. & 1 & Absent \\
\hline Meliaceae & Cedrela fissilis Vell. & 3 & Present \\
\hline Monimiaceae & Mollinedia clavigera Tul. & 1 & Absent \\
\hline Moraceae & Ficus luschnathiana Miq. & 2 & Present \\
\hline \multirow[t]{11}{*}{ Myrtaceae } & Calyptranthes concinna DC. & 1 & Absent \\
\hline & Campomanesia guaviroba (DC.) Kiaersk & 2 & Present \\
\hline & Campomanesia xanthocarpa O.Berg & 2 & Absent \\
\hline & Eugenia pluriflora Mart. & 2 & Absent \\
\hline & Eugenia pyriformis Cambess. & 2 & Present \\
\hline & Eugenia uniflora O.Berg & 2 & Absent \\
\hline & Myrceugenia miersiana (Gardner) D.Legrand \& Kausel & 1 & Absent \\
\hline & Myrcia cymoso-paniculata Kiaersk & 3 & Absent \\
\hline & Myrcia hatschbachii D.Legrand & 2 & Absent \\
\hline & Myrcia rostrata DC. & 3 & Present \\
\hline & Psidium cattleyanum Sabine & 1 & Absent \\
\hline Oleaceae & Chionanthus filiformis (Vell.) P.S.Green & 3 & Absent \\
\hline Picramniaceae & Picramnia parvifolia Engl. & 2 & Absent \\
\hline Piperaceae & Piper gaudichaudianum Kunth ex C.DC. & 1 & Absent \\
\hline Podocarpaceae & Podocarpus lambertii Klotzsch ex Endl. & 2 & Absent \\
\hline \multirow[t]{2}{*}{ Primulaceae } & Myrsine coriacea Nadeaud & 1 & Present \\
\hline & Myrsine umbellate Mart. & 1 & Absent \\
\hline Proteaceae & Roupala Montana Willd. & 2 & Absent \\
\hline \multirow[t]{2}{*}{ Rosaceae } & Prunus brasiliensis Schott ex Spreng. & 2 & Absent \\
\hline & Prunus sellowii Koehne & 2 & Absent \\
\hline \multirow[t]{2}{*}{ Salicaceae } & Casearia decandra Jacq. & 1 & Absent \\
\hline & Casearia sylvestris $\mathrm{Sw}$. & 1 & Absent \\
\hline \multirow[t]{4}{*}{ Sapindaceae } & Allophylus edulis Radlk. ex Warm. & 2 & Absent \\
\hline & Allophylus guaraniticus Radlk. & 2 & Absent \\
\hline & Cupania vernalis Cambess. & 1 & Absent \\
\hline & Matayba elaeagnoides Radlk. & 2 & Present \\
\hline \multirow[t]{3}{*}{ Solanaceae } & Solanum pseudoquina A.St.-Hil. & 1 & Absent \\
\hline & Solanum sanctae-catharinae Dunal & 2 & Absent \\
\hline & Solanum swartzianum Roem. \& Schult. & 2 & Absent \\
\hline Verbenaceae & Duranta vestita Cham. & 3 & Absent \\
\hline
\end{tabular}

heterobaric leaves of each forest type were compared using t-test. Both analyses were performed on PAST software (Hammer et al. 2001). The independent distribution analysis of heterobaric leaves among forests and strata employed the $\chi^{2}$ test $(P<0.05)$. Since MOF Stratum 4 included only one species, Araucaria angustifolia (Bertol.) Kuntze, it was excluded from this test, which aimed to verify the distribution of heterobaric leaves among plant families. The independent distribution analysis was performed using the RCMDR package $(2,1-7)$ for R program (version 3.1.2, R Foundation for Statistical computing, Vienna Austria).

\section{Results}

Of the 89 LLODF species studied, 22 (25\%), belonging to 10 families, had heterobaric leaves (Fig.1A-B), while
67 species (75\%) from 30 families had homobaric leaves (Fig.1C-D; Tab. 4). Out of the $58 \mathrm{MOF}$ species studied, 16 (28\%), belonging to 11 families, had heterobaric leaves, while 42 (72\%) from 26 families had homobaric leaves (Fig. 1A-B; Tab. 4). The proportion of species with each leaf type (homobaric/heterobaric) did not differ between the studied forests ( $\chi^{2}$ test, $P=0.75, \mathrm{GL}=1, \mathrm{~N}=146$ species). For MOF, all heterobaric leaves had sclerenchymatous BSEs (Fig. 1B), except Ficus luschnathiana and Myrsine coriacea, which had parenchymatous BSEs. In LLODF, all heterobaric leaves had sclerenchymatous BSE, and Gomidesia schaueriana had incomplete BSE; in this species, BSE occurred only on the adaxial side of the lamina.

The distribution of species with heterobaric leaves among forest strata differed significantly in LLODF ( $\chi 2$ test, $P=0.013, \mathrm{DF}=3, \mathrm{~N}=89$ species). The highest proportion of 

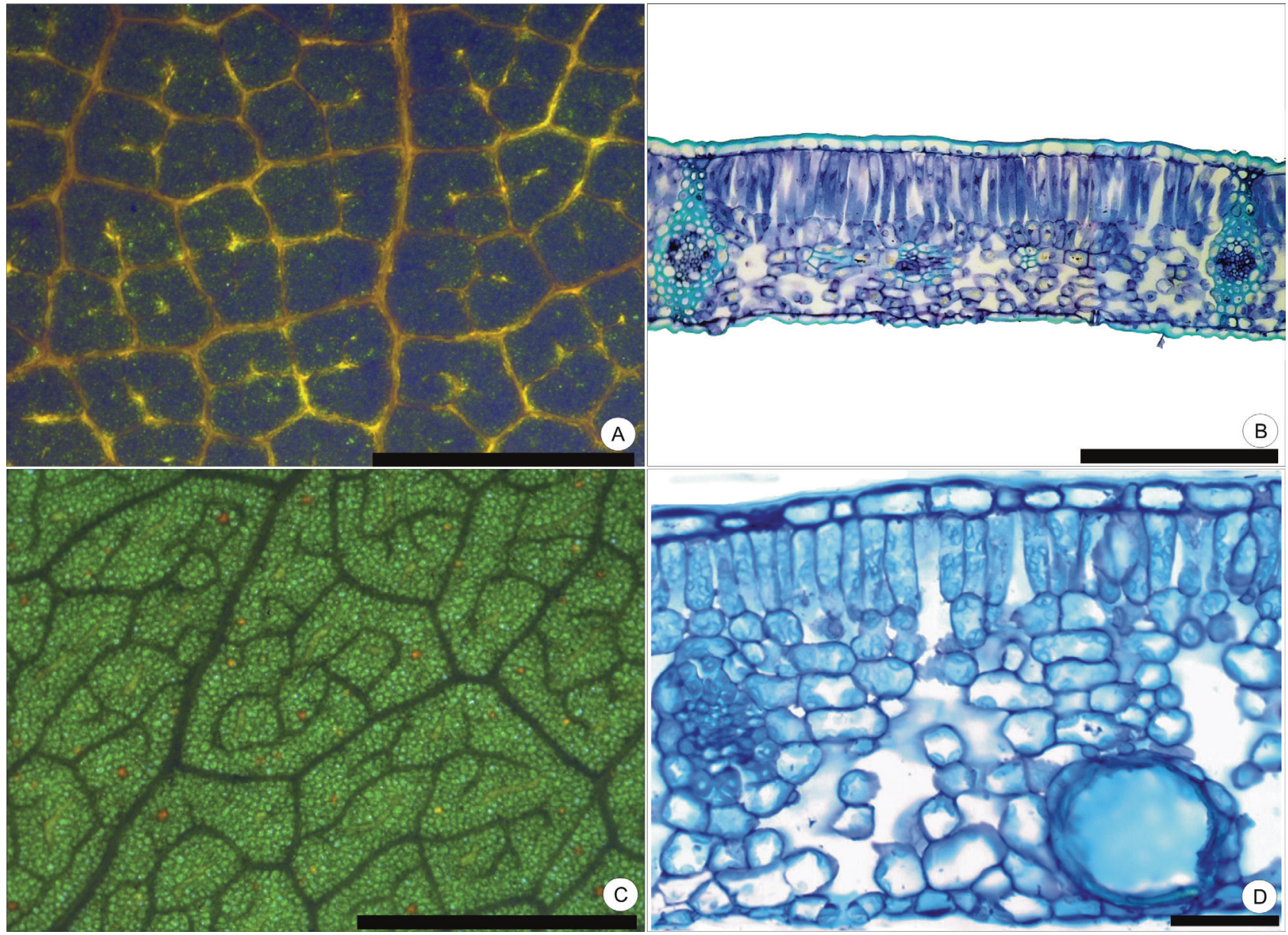

Figure 1. Surface and cross sections of homobric and heterobaric leaves. A. Surface of heterobaric leaf of Ocotea porosa, showing the division of the lamina in small areas by bundle sheath extension (BSE). B. Cross section of heterobaric leaf of Ocotea porosa, showing BSE. C. Surface of homobaric leaf of Lonchocarphus muehlbergianus, showing a homogeneous lamina. D. Cross section of homobaric leaf of Tibouchina sellowiana. Bars: A and C $=2 \mathrm{~mm} ; \mathrm{B}=30 \mu \mathrm{m} ; \mathrm{D}=50 \mu \mathrm{m}$

heterobaric leaves was found in Stratum 4 (Tab. 4), and the number of heterobaric leaves was directly proportional to light stratification. In MOF, despite the fact that heterobaric leaves were not significantly distributed among strata ( $\chi 2$ test, $P=0.0662, \mathrm{DF}=3, \mathrm{~N}=57$ species), an increase in heterobaric leaves was directly related to light intensity, with the highest proportion of heterobaric of leaves being in Stratum 3, since the frequency of heterobaric leaves was $0 \%$ in Stratum 4, which was composed of a single species (A. angustifolia) with only homobaric leaves. The lower strata had higher proportions of homobaric leaves in both forests (Tab. 4).

For all species considered in LLODF, leaf area did not differ among Strata 1, 2, and 3, but was higher in Stratum 4 (Tab. 5). When we excluded species with leaf area $>100 \mathrm{~cm}^{2}$, [Ormosia arborea in Stratum 1; Aparisthmium cordatum and Cupania oblongifolia in Stratum 2; Miconia cabucu in Stratum 3 and Coccoloba warmingii in Stratum 4], the average leaf area of Stratum 4 differed from Strata 2 and 3. Although these species occur in small numbers in each stratum, they significantly affected average leaf area, as shown by the standard deviations (Tab. 5). In MOF, there was no variation in leaf area among lower strata; only Stratum 4 differed due to the reduced leaf area of $A$. angustifolia leaves (Tab. 5).

In LLODF, leaf thickness did not exhibit the same pattern of variation among strata as leaf area. Only Stratum 3 differed by having thinner leaves than the other strata. When we excluded the species with leaf area $>100 \mathrm{~cm}^{2}$, mean leaf thickness varied as follows: Stratum $1=$ Stratum $2>$ Stratum $3>$ Stratum 4. In MOF, Stratum 4 had thicker leaves than the other strata (Tab. 5).

The comparison of homobaric and heterobaric leaves, independently of strata, indicates that homobaric leaves were thicker than heterobaric leaves in both forests types (LLODF $-\mathrm{t}$ test: $\mathrm{t}=19.65, P<0.001 ; \mathrm{MOF}-\mathrm{t}$ test $\mathrm{t}=18.79$, $P<0.001$ ), when leaves $>100 \mathrm{~cm}^{2}$ are excluded (Tab. 5).

The distribution of heterobaric leaves among some plant families was also evaluated ( $\chi^{2}$ test, $P<0.0001, \mathrm{GL}=7, \mathrm{~N}=$ 81 species from eight families with more than five species, Tab. S01 in supplementary material). In this study, all species of Lauraceae had heterobaric leaves, independently of strata (Fig. 2), except Endlicheria paniculata, which had heterobaric 
Maria Regina Torres Boeger, Maiara Matilde Silva,

Guilherme Nogueira, Allan Alvarenga and Suellen Silva Pereto

Table 4. Number and percentage of species with homobaric and heterobaric leaves by forest type and stratum. Legend: LLODF Lowland Ombrophilous Dense Forest; MOF - Mixed Ombrophilous Forest.

\begin{tabular}{|c|c|c|c|c|c|}
\hline Forest type & Stratum & $\begin{array}{l}\mathrm{N}^{\circ} \text { of species with } \\
\text { heterobaric leaves }\end{array}$ & $\begin{array}{l}N^{0} \text { of species with } \\
\text { homobaric leaves }\end{array}$ & Total & $\begin{array}{c}\text { Species with } \\
\text { heterobaric leaves } \\
{[\%]}\end{array}$ \\
\hline \multirow[t]{4}{*}{ LLODF } & $1(<5 \mathrm{~m})$ & 0 & 21 & 21 & 0 \\
\hline & $2(5.0-9.9 \mathrm{~m})$ & 3 & 35 & 38 & 7.9 \\
\hline & $3(10-14.9 m)$ & 5 & 9 & 14 & 35.7 \\
\hline & $4(>15 \mathrm{~m})$ & 14 & 2 & 16 & 87.5 \\
\hline Total & & 22 & 67 & 89 & 25.0 \\
\hline \multirow[t]{4}{*}{ MOF } & $1(<7 \mathrm{~m})$ & 3 & 18 & 21 & 14.3 \\
\hline & $2(7-14.9 m)$ & 4 & 19 & 23 & 17.4 \\
\hline & $3(15-26 m)$ & 9 & 4 & 13 & 69.2 \\
\hline & $4(>26 m)$ & 0 & 1 & 1 & 0 \\
\hline Total & & 16 & 42 & 58 & 28 \\
\hline
\end{tabular}

Table 5. Average height, average values and respective standard deviations of leaf area and leaf thickness by stratum, leaf types and forest type. Legend: LLODF - Lowland Ombrophilous Dense Forest; MOF - Mixed Ombrophilous Forest. $\left(^{*}\right)$ Leaf area and leaf thickness averages with the exclusion of leaves $>100 \mathrm{~cm} 2$, only in LLODF. Different letters in the same column, within the forest type, represent statistical difference, Tukey test $(\mathrm{p}<0.05)$.

\begin{tabular}{|c|c|c|c|c|c|c|}
\hline Forest type & Stratum & Height [m] & Leaf area $\left[\mathrm{cm}^{2}\right]$ & Leaf area $*\left[\mathrm{~cm}^{2}\right]$ & Leaf thickness (mm) & Leaf thickness $(\mathrm{mm}]^{*}$ \\
\hline \multirow[t]{4}{*}{ LLODF } & $1(<5 \mathrm{~m})$ & $3.4(1.4)$ & $29.3(25.1) \mathrm{b}$ & $23.1(11.0) a b$ & $0.31(0.1) a$ & $0.30(0.1) a$ \\
\hline & $2(5-9.9 m)$ & $7.5(1.5)$ & $30.5(25.4) \mathrm{b}$ & $27.5(19.6) \mathrm{a}$ & $0.32(0.2) \mathrm{a}$ & $0.32(0.2) \mathrm{a}$ \\
\hline & $3(10-14.9 \mathrm{~m})$ & $11.7(1.1)$ & $35.3(56.0) \mathrm{b}$ & $19.1(11.2) \mathrm{b}$ & $0.23(0.1) b$ & $0.21(0.1) b$ \\
\hline & $4(>15 m)$ & $16.3(1.6)$ & $81.5(81.3) \mathrm{a}$ & $25.4(30.1) \mathrm{a}$ & $0.31(0.1) \mathrm{a}$ & $0.16(0.1) c$ \\
\hline Homobaric leaves & all & - & $32.5(35.6) \mathrm{a}$ & $15.0(11.9) \mathrm{b}$ & $0.30(0.15) \mathrm{a}$ & $0.31(0.16) \mathrm{a}$ \\
\hline Heterobaric leaves & all & - & $39.6(46.3) \mathrm{a}$ & $19.6(14.0) \mathrm{a}$ & $0.30(0.12) \mathrm{a}$ & $0.29(0.10) \mathrm{b}$ \\
\hline \multirow[t]{4}{*}{ MOF } & $1(<7 \mathrm{~m})$ & $5.2(1.8)$ & $14.7(12.3) \mathrm{a}$ & & $0.18(0.1) b$ & \\
\hline & $2(7-14.9 \mathrm{~m})$ & $9.2(3.3)$ & $18.3(13.4) \mathrm{a}$ & & $0.19(0.1) \mathrm{b}$ & \\
\hline & $3(15-26 \mathrm{~m})$ & $12.7(9.2)$ & $16.2(11.1) \mathrm{a}$ & & $0.18(0.0) b$ & \\
\hline & $4(>26 \mathrm{~m})$ & $30.1(12.0)$ & $1.04(0.2) \mathrm{b}$ & & $0.36(0.07) \mathrm{a}$ & \\
\hline Homobaric leaves & all & - & $15.0(11.9) \mathrm{a}$ & & $0.20(0.08) \mathrm{a}$ & \\
\hline Heterobaric leaves & all & - & $19.6(14.1) \mathrm{a}$ & & $0.15(0.04) \mathrm{b}$ & \\
\hline
\end{tabular}

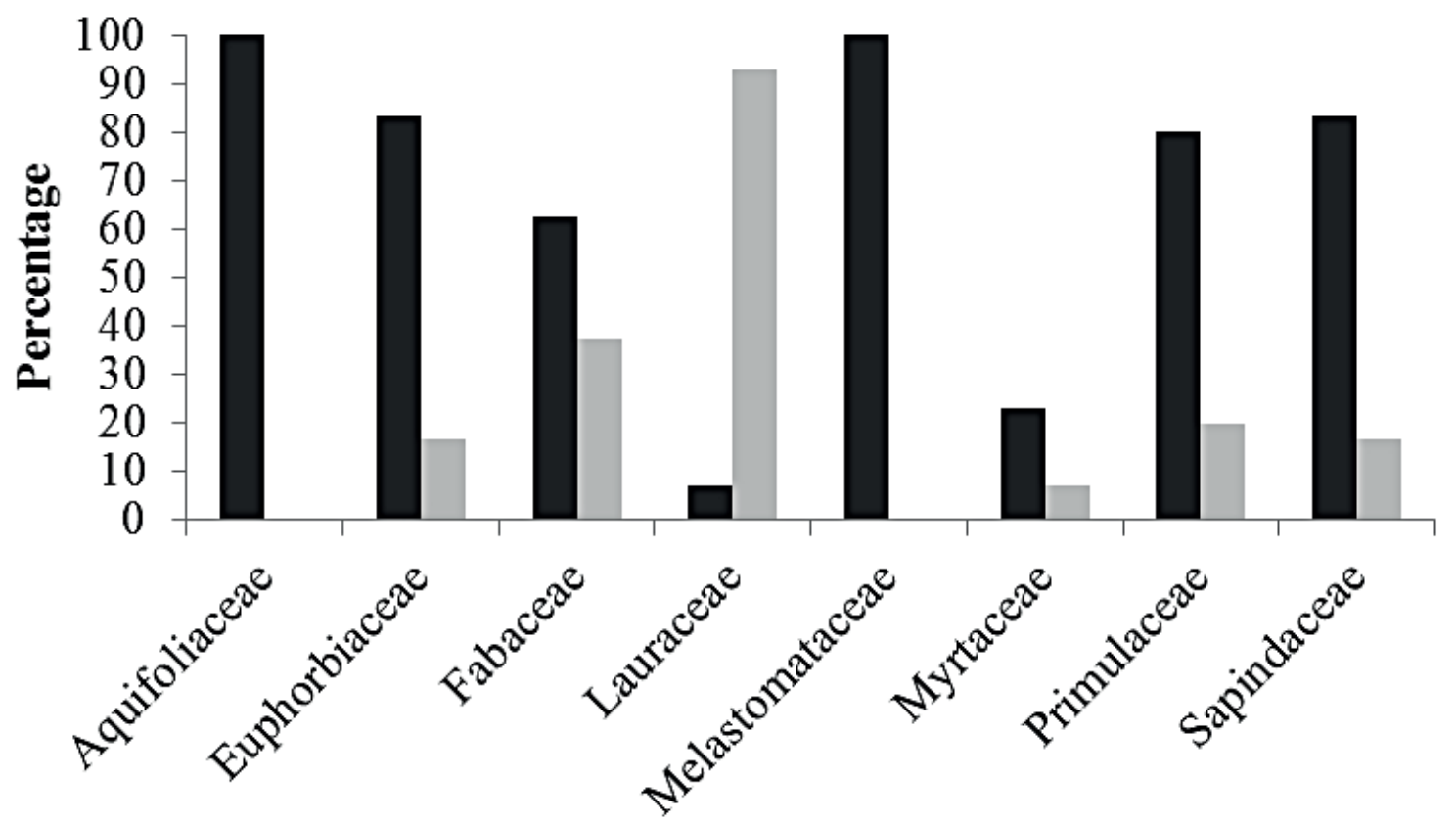

Figure 2. Percentage of homobaric and heterobaric leaves among plant families with more than five species. Black bars represent homobaric leaves and grey bars represent heterobaric leaves. 


\section{Occurrence of homobaric and heterobaric leaves in two forest types of southern Brazil}

leaves in Stratum 1 at LLODF.

Besides Lauraceae, Euphorbiaceae, Fabaceae, Myrtaceae, Primulaceae, and Sapotaceae also had species with both leaf types, with heterobaric leaves being mainly distributed in Strata 2 and 3. No species of Aquifoliaceae and Melastomataceae, which occurred mainly in Strata 1 and 2 , had heterobaric leaves.

\section{Discussion}

Both forest types exhibited similar proportions of species with homobaric and heterobaric leaves, with a greater occurrence of the former. Heterobaric leaves are generally associated with deciduous forests with cold or dry, well-defined seasons (Terashima 1992). On the other hand, homobaric leaves occur in evergreen forests, generally found in humid and hot regions (Kenzo et al. 2007). Thus, our data corroborate a previous study that found a higher proportion of homobaric leaves in humid forests with high precipitation throughout the year (Kenzo et al. 2007), such as our study sites. Although heterobaric leaves are associated with drier, deciduous forests, they are also present in humid forests, such as was found in our study sites. The distribution of these two types of leaves among strata in the present study was similar to that observed by Kenzo et al. (2007) in a rain forest at Sarawak, Malasia.

The distribution analysis indicated that heterobaric leaves are more common in Strata 3 and 4 in MOF and LLODF, respectively, while homobaric leaves were more common in Strata 1 and 2. This distribution of homobaric and heterobaric leaves in different strata appears to be due to micro-environmental gradients associated with the various forest strata (Kenzo et al. 2007). Such gradients include light availability, temperature, vapor pressure deficit, and wind (Théry 2001; Kitajima \& Poorter 2010; Bennett et al. 2015; Inoue et al. 2015). Of these, light availability is particularly important because it can influence the growth, survival, and subsequent reproduction of young individuals (Chazdon et al. 1996; Valladares \& Niinemets 2008).

In the canopy, for example, plants are subjected to intense light and heat, which can be stressful during some periods of the day and/or year (Théry 2001; Valladares \& Niinemets 2008). The presence of heterobaric leaves in the higher strata of a forest can be advantageous because sclerenchymatous BSEs can give additional mechanical support, due to the strength given by the sclerenchyma cells (Dickson 2000; Cutler et al. 2008), and help to maintain leaf shape and volume (Roth 1984), as well as protect against herbivores (Sack \& Scoffoni 2013). Secondarily, BSEs can perform optic functions such as facilitating the dispersion of light within the compartments of the leaf (Karabourniotis et al. 2000; Nikolopoulos et al. 2002), thereby enhancing photosynthetic rate (Nikolopoulos et al. 2002; Liakoura et al. 2009; Buckley et al. 2011).

Plants restricted to lower strata, on the other hand, are subjected to low levels of heterogeneous light (Théry 2001; Kenzo et al. 2007; Valladares \& Niinemets 2008). These conditions are beneficial to homobaric leaves with their welldeveloped spongy parenchyma (Fig. 1D), as observed in the studied species [spongy:palisade parenchyma ratio for MOF homobaric leaves ( $2.1 \pm 0.9)$; for MOF heterobaric leaves (1.5 $\pm 0.6)$; for LLODF homobaric leaves (5.3 \pm 3.2$)$ and for LLODF heterobaric leaves $(3.9 \pm 1.7)]$. A thicker spongy parenchyma is advantageous for capturing diffused light because the irregular-shaped cells reflect light rays within the mesophyll, thereby facilitating more efficient absorption (Vogelmann et al. 1996). Homobaric leaves also increase the proportion of photosynthetic areas in the mesophyll (Terashima 1992), which may contribute to more efficient photosynthesis and water use (Pieruschka et al. 2006; Pieruschka et al. 2010; Lynch et al. 2012) Thus, under limited light conditions, species with homobaric leaves perform better than those with heterobaric leaves (Kenzo et al. 2007).

The distribution of species with homobaric and heterobaric leaves was weakly correlated with taxonomic group. Although Lauraceae is present in all strata of LLODF and in the first three strata of MOF, it is the only family that is represented by a larger number of heterobaric leaf species. All the species of the families Aquifoliaceae and Melastomataceae, which are commonly found in under-canopy strata, had homobaric leaves. Even though they comprise species with both leaf types, the families Euphorbiaceae, Myrtaceae, Primulaceae, and Sapindaceae did not show a distributional pattern related to strata. The one exception was Fabaceae, whose species with heterobaric leaves were present in Stratum 3 in MOF. These results indicate that the leaf types of each species are more dependent on habitat and/or life form type than phylogenetic relationships. Environmental filters have convergent effects and seem to favor functional diversity due the habitat heterogeneity, especially in tropical forest communities (Manel et al. 2014).

In conclusion, the occurrence of homobaric and heterobaric leaves seems to be related to light stratification. The distribution of homobaric and heterobaric leaves in the different forest strata shows that light stratification acts as an ecological filter on the composition of the vegetation. Heterobaric leaves tend to occur in hotter strata that are more exposed to light, while homobaric leaves are more frequent in the under-canopy and more humid strata. This difference indicates that both leaf types occupy different positions on the "leaf economic spectrum", based on the balance between the cost of investiment in structural tissues and the investiment in photosynthetic tissues for carbon fixation via photosynthesis (sense Wright et al. 2004).

Besides environmental influences, the occurrence of leaf types is weakly related to taxonomic group. Only Lauraceae included a large number of heterobaric species. These results show that these two leaf types (homobaric/heterobaric) are more dependent on habitat and/or life form than phylogenetic relationships. Environmental filters seem to shape functional diversity due to habitat heterogeneity, especially in tropical forest communities. 


\section{Acknowledgements}

The authors are grateful to the "Coordenação de Aperfeiçoamento de Pessoal de Nível Superior" (CAPES) for scholarship to the co-authors and to the productivity fellowship to the first author (process $n^{\circ} 301971 / 2013-7$ ).

\section{References}

Bennett AC, McDowell NG, Allen CD, Anderson-Teixeira KJ. 2015. Larger trees suffer most during drought in forest worldwide. Nature Plants 1: 15139. doi:10.1038/nplants.2015.139.

Boeger MRT, Alves LC, Negrelle RRB. 2004. Leaf morphology of 89 tree species from a lowland tropical rain forest (Atlantic forest) in South Brazil. Brazilian Archives of Biology and Technology 47: 933-943.

Buckley TN, Sack L, Gilbert ME. 2011. The role of bundle sheath extensions and life form in stomatal responses to leaf water status. Plant Physiology 156: 962-973.

Chazdon RL, Pearcy RW, Lee DW, Fetcher N. 1996. Photosynthetic responses of tropical forests plants to contrasting light environments. In: Mulkey SS, Chazdon RL, Smith AP. (eds.) Tropical Forest Ecophysiology. New York, Chapman and Hall. p. 5-55.

Cutler DF, Botha T, Stevenson DW. 2008. Plant Anatomy - an applied approach. Malden, Blackwell Publishing.

Dickson WC. 2000. Integrative plant anatomy. San Diego, Academic Press.

Hammer O, Harper DAT, Ryan PD. 2001. PAST: Palaeontological statistics software package for education and data analysis. Palaeontologia Eletronica 4: 9.

Inoue Y, Kenzo T, Tanaka-Oda A, Yoneyama A, Ichie T. 2015. Leaf water use in heterobaric and homobaric leafed canopy tree species in a Malaysian tropical rain forest. Photosynthetica 53: 177-186.

Karabourniotis G. 1998. Light-guiding function of foliar sclereids in the evergreen sclerophyll Phillyrea latifolia: a quantitative approach. Journal of Experimental Botany 49: 739-746.

Karabourniotis G, Bornman JF, Nikolopoulos D. 2000. A possible optical role of the bundle sheath extensions of the heterobaric leaves of Vitis vinifera and Quercus coccifera. Plant, Cell and Environment 23: 423-430.

Kenzo T, Ichie T, Watanabe Y, Hiromi T. 2007. Ecological distribution of homobaric and heterobaric leaves in tree species of Malaysian lowland tropical rainforest. American Journal of Botany 94: 764-775.

Kitajima K, Poorter L. 2010. Tissue-level leaf toughness, but not lamina thickness, predicts sapling leaf lifespan and shade tolerance of tropical tree species. New Phytologist 186: 708-721.

Liakoura V, Fotelli MN, Rennenberg H, Karabourniotis G. 2009. Should structure -function relations be considered separately for Homobaric vs. Heterobaric leaves? American Journal of Botany 96: 612-619.
Lynch DJ, McInerney FA, Kouwenberg LL, Gonzalez-Meler MA. 2012. Plasticity in bundle sheath extensions of heterobaric leaves. American Journal of Botany 99: 1197-1206.

Manel S, Couvreur TLP, Munoz F, Couteron P, Hardy OJ. 2014. Characterizing the phylogenetic tree community structure of a protected tropical rain forest area in Cameroon. PLoS ONE 9(6): e98920. doi:10.1371/ journal.pone.0098920

Niinements Ü. 2010. A review of light interception in plant stands from leaf to canopy in different plant functional types and in species with varying shade tolerance. Ecological Research 25: 693-714.

Nikolopoulos D, Liakopoulos G, Drossopoulos I, Karabourniotis G. 2002. The relationship between anatomy and photosynthetic performance of heterobaric leaves. Plant Physiology 129: 235-243.

Pieruschka R, Schuur U, Jensen M, Wolff WF, Jahnke S. 2006. Lateral diffusion of $\mathrm{CO}_{2}$ from shaded to illuminate leaf parts affects photosynthesis inside homobaric leaves. New Phytologist 169: 779788.

Pieruschka R, Chavarría-Krauser A, Schurr U, Jahnke S. 2010. Photosynthesis in lightfleck areas of homobaric and heterobaric leaves. Journal of Experimental Botany 61: 1031-1039.

Rhizopoulou S, Psaras G. 2003. Development and structure of droughttolerant leavesof the Mediterranean shrub Capparis spinosa L. Annals of Botany 92: 377-383.

Roth I. 1984. Stratification of tropical forests as seen in leaf structure. Tasks for vegetation Science. Vol. 6. Hague-Boston-Lancaster, Dr. W. Junk Publishing.

Sack L, Scoffoni C. 2013. Leaf venation: structure, function, development, evolution, ecology and applications in the past, present and future. New phytologist 198: 983-1000.

Silveira TA, Boeger MRT, Maranho LT, Melo Jr. JCF, Soffiatti P. 2015. Functional leaf traits of 57 woody species of the Araucaria Forest, Southern Brazil. Brazilian Journal of Botany 38: 357-366.

Terashima I. 1992. Anatomy of non-uniform leaf photosynthesis. Photosynthesis Research, 31: 195-212. doi: 10.1007/BF00035537.

Théry M. 2001. Forest light and its influence in habitat selection. Plant Ecology 153: 251-261.

Turner IM. 1994. A quantitative analysis of leaf form in woody plants from the world's major broad leaved forest types. Journal of Biogeography 21: 413-419.

Valladares F, Niinemets Ü. 2008. Shade tolerance, a key plant feature of complex nature and consequences. Annual Review of Ecology, Evolution and Systematics 39: 237-257.

Vogelman TC, Nishio JN, Smith WK. 1996. Leaves and light capture: light propagation and gradients of carbon fixation within leaves. Trends Plant Science 1: 65-70.

Wright IJ, Reich PB, Villar R. 2004. The worldwide leaf economics spectrum. Nature 428: 821-827.

Zwieniecki MA, Brodribb TJ, Holbrook NM. 2007. Hydraulic design of leaves: insights from rehydration kinetics. Plant Cell Environment 30: 910-921. 